\title{
Renal effects of treatment with a TLR4 inhibitor in conscious septic sheep
}

\author{
Johan Fenhammar ${ }^{1}$, Mats Rundgren ${ }^{2}$, Kjell Hultenby ${ }^{3}$, Jakob Forestier ${ }^{1}$, Micael Taavo ${ }^{4}$, Ellinor Kenne ${ }^{5,6}$, \\ Eddie Weitzberg ${ }^{2}$, Stefan Eriksson ${ }^{2}$, Volkan Ozenci ${ }^{7}$, Annika Wernerson ${ }^{8}$ and Robert Frithiof ${ }^{2,4^{*}}$
}

\begin{abstract}
Introduction: Acute kidney injury (AKI) is a common and feared complication of sepsis. The pathogenesis of sepsis-induced AKI is largely unknown, and therapeutic interventions are mainly supportive. In the present study, we tested the hypothesis that pharmacological inhibition of Toll-like receptor 4 (TLR4) would improve renal function and reduce renal damage in experimental sepsis, even after AKI had already developed.

Methods: Sheep were surgically instrumented and subjected to a 36-hour intravenous infusion of live Escherichia coli. After 12 hours, they were randomized to treatment with a selective TLR4 inhibitor (TAK-242) or vehicle.

Results: The E. coli caused normotensive sepsis characterized by fever, increased cardiac index, hyperlactemia, oliguria, and decreased creatinine clearance. TAK-242 significantly improved creatinine clearance and urine output. The increase in N-acetyl-beta-D-glucosaminidas, a marker of tubular damage, was attenuated. Furthermore, TAK-242 reduced the renal neutrophil accumulation and glomerular endothelial swelling caused by sepsis. These effects were independent of changes in renal artery blood flow and renal microvascular perfusion in both cortex and medulla. TAK-242 had no effect per se on the measured parameters.

Conclusions: These results show that treatment with a TLR4 inhibitor is able to reverse a manifest impairment in renal function caused by sepsis. In addition, the results provide evidence that the mechanism underlying the effect of TAK-242 on renal function does not involve improved macro-circulation or micro-circulation, enhanced renal oxygen delivery, or attenuation of tubular necrosis. TLR4-mediated inflammation resulting in glomerular endothelial swelling may be an important part of the pathogenesis underlying Gram-negative septic acute kidney injury.
\end{abstract}

\section{Introduction}

Acute kidney injury (AKI) is an important contributor to morbidity and mortality among hospitalized patients. Sepsis is the leading cause of AKI in the critically ill patient, and currently no effective treatment exists [1]. Septic AKI is generally believed to be due to regional hypoperfusion causing renal ischemia [2]. However, recent experimental reports indicate that AKI may develop even though renal blood flow and blood pressure remain within physiological limits [3-5]. Thus, it is possible that the pathogenesis of septic AKI is complicated by factors other than ischemia. In line with this, we wanted to

\footnotetext{
* Correspondence: robert.frithiof@surgsci.uu.se

${ }^{2}$ Department of Physiology and Pharmacology, Karolinska Institutet, von Eulers väg 8, 17177 Stockholm, Sweden

${ }^{4}$ Department of Surgical Sciences, Section of Anaesthesiology \& Intensive Care, Uppsala University, Sjukhusvägen 1, 75185 Uppsala, Sweden Full list of author information is available at the end of the article
}

investigate whether an important receptor for initiating inflammation, the Toll-like receptor 4 (TLR4), participates in the pathophysiology of Escherichia coli-induced AKI. TLRs have been identified as pivotal mediators in host defense as they are crucial in pathogen recognition and activation of the immune response [6,7]. The main ligand for TLR4 is lipopolysaccharide (LPS), a component of the cell membrane of Gram-negative bacteria [8-10]. When LPS binds to TLR4, an inflammatory response is initiated via production and release of cytokines as well as stimulation of inflammatory cells [11]. Targeting TLR4 with antibodies or specific inhibitors has shown beneficial effects by reducing mortality in experimental models of Gram-negative sepsis [12-17]. Initial clinical trials also showed beneficial effects of blocking TLR4 in sepsis [18], but in larger follow-up investigations, survival was not improved $[19,20]$. However, the renal effects of TLR4 antagonism are incompletely 
investigated, and there are reasons to believe that TLR4 may be an important mediator of sepsis-induced AKI. For example, the expression of TLR4 in renal tubules, glomeruli, and peri-tubular capillaries is increased after sepsis [21], and mice deficient in TLR4 have a reduced increase in blood urea nitrogen (BUN) when subjected to LPS [22]. Our group recently showed that pretreatment with TAK-242 attenuated oliguria and reduced creatinine clearance in LPS-induced hyperdynamic shock [3]. However, although the aforementioned results are promising, the potential of TLR4 modulation during sepsis and septic renal failure, as well as mechanisms of action, is still largely unknown.

One of the main objectives with the current study was to investigate whether TLR4 inhibition is effective in attenuating or reversing renal dysfunction even after sepsis has developed. Therefore, TAK-242 was administered 12 hours into normotensive ovine sepsis caused by an intravenous live $E$. coli infusion, and the effect on renal function was followed for an additional 24 hours. To further explore the hypothesis that septic AKI is due to regional blood flow restriction, total renal blood flow and cortical and medullary microcirculation were continuously measured. Signs of ischemia were repeatedly monitored by microdialysis in the cortex and medulla. It is highly debatable whether septic AKI is associated with renal histopathology, and studies investigating structural damage to the kidney in long-term animal model of sepsis are lacking [23]. Thus, both light and electron microscopy were used to analyze renal samples taken at the end of the 36-hour study period with regard to local injury and leukocyte infiltration.

\section{Materials and methods}

For detailed description of methods, please refer to the online supplemental material (Additional file 1). The experiments conform to the guidelines laid out in the Guide for the Care and Use of Laboratory Animals (National Academy of Science). The Regional Ethics Committee for Experiments in Animals, Stockholm, Sweden, approved the study in advance (N285/08).

\section{Surgical preparation and study protocol}

Twenty-seven adult Texel crossbred ewes were included in the study. Twenty-four of these sheep were anesthetized and prepared with catheters in the right carotid artery, the pulmonary artery, and the right jugular vein. For renal hemodynamic measurements, an ultrasonic flow probe was placed around the left renal artery, and two laser Doppler flow probes, one cortical and one medullar, were sutured on the left kidney. Intrarenal metabolism was studied by microdialysis catheters in the cortex and in the medulla, respectively. A urinary retention catheter was inserted into the bladder for urine sampling. After a post-surgical recovery period of 12 to 18 hours, the experiments commenced with the animals being conscious and placed in a pen. Sepsis was induced by an intravenous infusion of live $E$. coli bacteria (bolus of $3.9 \times 10^{9}$ colony-forming units followed by an infusion of $6.0 \times 10^{9} / \mathrm{mL}$ colony-forming units, starting at a rate of $0.2 \mathrm{~mL} /$ hour). The infusion rate was increased stepwise every 6 hours until reaching $4 \mathrm{~mL} /$ hour after 30 hours. After 12 hours of sepsis, sheep were randomized to receive a bolus dose $(2 \mathrm{mg} / \mathrm{kg})$ followed by a continuous infusion ( $4 \mathrm{mg} / \mathrm{kg}$ per 24 hours) of either the selective TLR4 inhibitor TAK-242 $(10 \mathrm{mg} / \mathrm{mL})(\mathrm{n}=7)$ or vehicle $(n=7)$. The treatment was blinded to the investigators, and the content of the infusions was revealed only after the experiments were performed. To exclude that surgery per se had a major impact on the results obtained in the TAK-242 and vehicle groups, an additional sheep served as time control. This included surgical preparation and recovery and monitoring for 36 hours but no E. coli infusion or treatment.

To investigate whether TAK-242 had any effect on renal function per se, an additional three sheep were surgically prepared and, in a cross-over design, subjected to treatment with either TAK-242 or vehicle. The treatment was initiated by a bolus dose $(2 \mathrm{mg} / \mathrm{kg})$ followed by a continuous infusion ( $2 \mathrm{mg} / \mathrm{kg}$ per 12 hours). No E. coli was administered.

Besides the intravenous fluids given post-surgery, fluid volume support was administered as Ringer's Acetate solution (B. Braun Melsungen, AG, Melsungen, Germany) intravenously at $1 \mathrm{~mL} / \mathrm{kg}$ per hour and started 6 hours before the infusion of live $E$. coli bacteria. Blood samples (approximately $20 \mathrm{~mL}$ of venous blood and $1 \mathrm{~mL}$ of arterial blood) were drawn at baseline and every 6 hours after commencement of sepsis. Urinary output was measured and urine samples were collected every second hour. After 36 hours of sepsis, animals were deeply anaesthetized with sodium thiopental and terminated by an overdose of potassium chloride. The kidney was rapidly harvested and prepared for histological evaluation. The position of the laser Doppler probes and microdialysis catheters was confirmed visually by opening the kidney post-mortem. Renal biopsies were immediately frozen and stored at $-70^{\circ} \mathrm{C}$. If the animal was judged to be severely ill and in distress, it was euthanized prior to the end of the protocol.

\section{Histological evaluation of renal biopsies}

Small pieces of renal tissue were prepared and evaluated by light and transmission electron microscopy. In addition, some sections were stained with anti-myeloperoxidase antibody for easy quantification of polymorphonuclear leukocyte (PMN) cells in the glomeruli, the interstitium, and the peritubular capillaries. 


\section{Statistical analysis}

Cardiovascular parameters were averaged off-line. Creatinine clearance was calculated as (urine flow $\times$ urine creatinine concentration)/plasma creatinine concentration. Cardiac output was indexed to body surface area $\left(0.09 \times\right.$ body weight $\left.{ }^{0.67}\right)$.

All statistical calculations were performed by using Statistica 8.0 (Statsoft Inc., Tulsa, OK, USA), and the graphs were created with Sigma Plot 11.0 (SPSS Inc., Chicago, IL, USA). Data are expressed as means \pm standard deviation of the mean or as mean and $95 \%$ confidence interval. Urine production, fractional sodium excretion (FENa), and urinary- $\mathrm{N}$-acetyl-beta- $\mathrm{D}$-glucosaminidase (U-NAG) values were transformed to follow a normal distribution by taking the logarithm of the raw data. Changes in parameters over time were analyzed according to a two-way repeated measures analysis of variance (ANOVA), with time as within effects and treatment (control/TAK-242) as between effects. If there was a significant interaction between time $\times$ treatment, an additional one-way repeated measures ANOVA was performed for each treatment to investigate whether that group changed significantly over time. The result of this analysis is not displayed in the figures but is referred to in the Results section. The significance level was set at a $P$ value of not more than 0.05 . Mann-Whitney $U$ test was used to evaluate difference in PMN count and histological scores between treatments.

\section{Results}

Nine out of twenty-three sheep subjected to E. coli had to be euthanized before completion of the protocol: five before treatment was started as well as two TAK-242treated animals and two vehicle-treated animals between 12 and 14 hours of sepsis. These sheep are not included in the data presented in the figures/tables or the statistical analyses. No effect of surgery per se was seen in the sheep that was sham-operated (Additional file 2: Table S3).

\section{E. coli infusion caused hyperdynamic sepsis}

Intravenous infusion of live $E$. coli bacteria caused classic signs of hyperdynamic sepsis; tachycardia, pulmonary hypertension, increased cardiac index, and a decrease in systemic vascular resistance (Figure $1 \mathrm{~A}-\mathrm{C}$ and F, $P<0.05$ for all). However, mean arterial blood pressure remained close to baseline levels, indicating that the animals were not in shock (Figure 1E). TAK-242 attenuated the increase in mean pulmonary arterial pressure (MPAP) but had no significant effect on any other of these variables (Figure 1). Arterial levels of lactate increased as a response to sepsis. However, in parallel with an improved renal function, TAK-242 reversed this trend and reduced lactate levels to near baseline values
(Figure 1D). Treatment with TAK-242 attenuated the decline in arterial partial pressure of oxygen $\left(\mathrm{pO}_{2}\right)$ caused by sepsis and it ended significantly higher compared with the control group. Partial pressure of carbon dioxide $\left(\mathrm{pCO}_{2}\right)$ increased in both groups without intergroup difference. No significant change over time or difference between the groups was observed in arterial $\mathrm{pH}$, base excess, hematocrite, plasma protein, sodium, or potassium levels (Additional file 3: Table S1). Sepsis induced nitric oxide (NO) formation as indicated by increased plasma levels of NOx (sum of nitrite and nitrate) and cyclic guanosine monophosphate $(P<0.05$ for both) with no effect of treatment with TAK-242. NOx in urine did not change significantly during the experiment (Additional file 3: Table S1).

\section{Sepsis-induced renal dysfunction was reversed by the TLR4 inhibitor TAK-242}

After 12 hours of sepsis, renal function was reduced as measured by elevated plasma levels of creatinine and $\mathrm{BUN}$, together with a decrease in creatinine clearance, urine production, and filtration fraction (Figure 2A-E, $P<0.05$ for all). The TLR4 inhibitor had a clear beneficial effect on renal function: Urine production decreased progressively in the control group, reaching severe oliguria in all animals, but TAK-242 not only prevented this decline but also improved diuresis significantly between 12 and 36 hours $(P<0.01$, Figure $2 \mathrm{~A})$. Furthermore, treatment with TAK-242 abolished the impairment in creatinine clearance (Figure 2, $P=0.002$ ) and attenuated the increase in plasma levels of creatinine, filtration fraction, and $\operatorname{BUN}(P<0.05$, for all Figure 2$)$. U-NAG, a marker of tubular damage, was significantly elevated at the end of the experiment, but TAK-242 attenuated this increase $(P<0.05$, Figure $2 \mathrm{~F})$, indicating less tubular injury in sheep with TLR4 inhibition.

\section{Sepsis did not cause renal hypoperfusion}

A possible explanation for the beneficial effects of TAK242 was prevention of renal hypoperfusion. However, renal artery blood flow increased $(P<0.001)$ during sepsis and renal vascular resistance was significantly decreased $(P<0.001)$ without any intergroup differences (Figure 3). This was mirrored as an increased perfusion in the renal cortex $(P<0.05)$, but medullary perfusion remained close to baseline levels in both groups (Figure 3). Light microscopy revealed no obvious renal tubular damage that explained the renal dysfunction. The tubules appeared normal except in two of the vehicle animals, where areas of proximal tubular injury were seen within the cortex (Figure 4). In such areas, the epithelial cells showed pyknotic or disintegrated nuclei (Figure 4D) or isometric vacuolization of the cytoplasm or both. In one of the TAK-242-treated animals, similar but much milder 


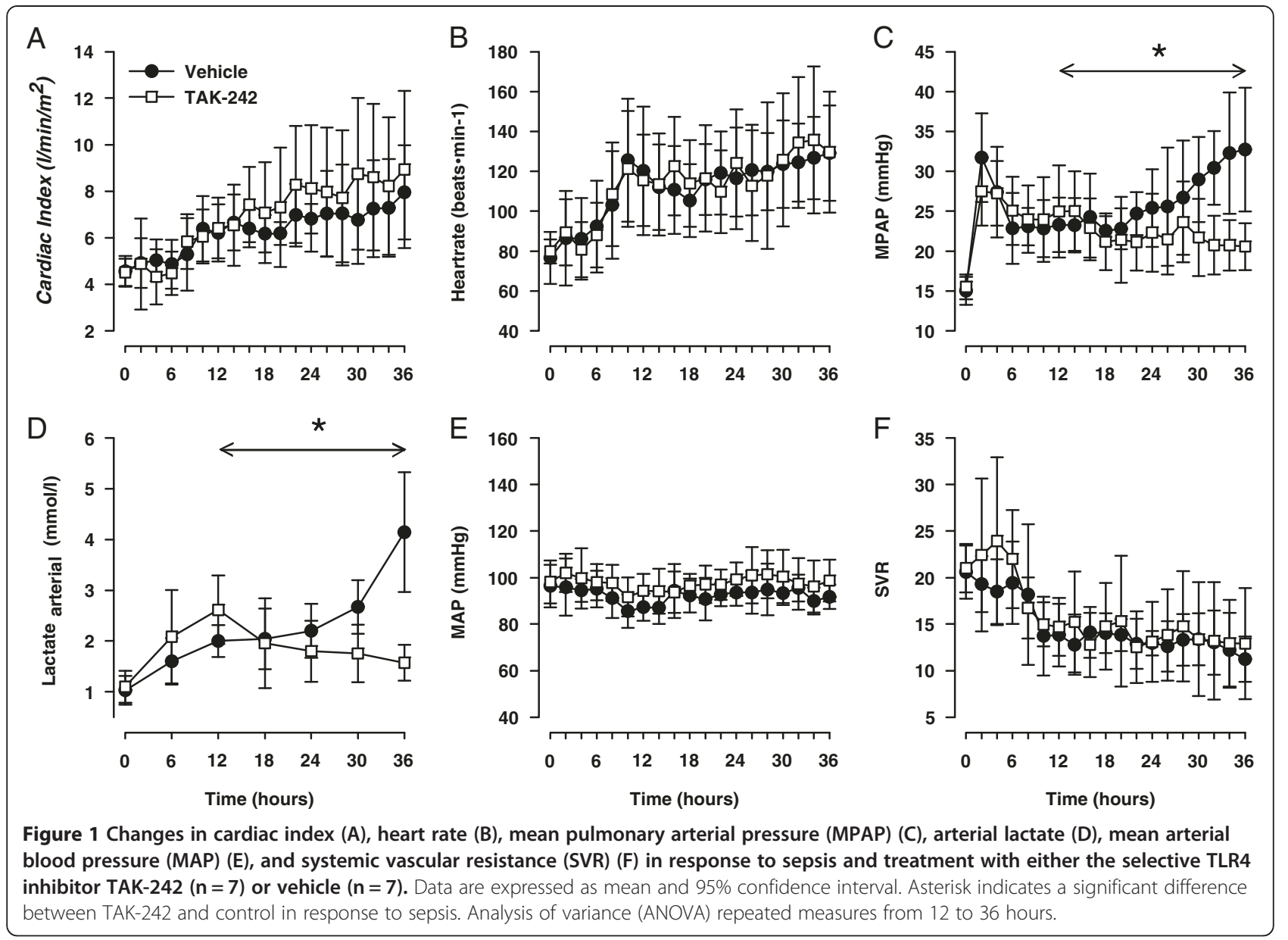

changes were seen. No changes were seen in larger vessels. In two of the vehicle animals, fibrin thrombi were found in the glomerular capillaries (Additional file 4: Figure S1).

\section{Treatment with a TLR4 inhibitor attenuated septic renal hyperlactemia}

To measure renal metabolic changes, microdialysis probes were placed in the renal cortex and medulla. During the first 12 hours of sepsis lactate, pyruvate and the lactate/pyruvate $(\mathrm{L} / \mathrm{P})$ ratio increased in both the cortex and the medulla whereas glucose was not significantly altered (Additional file 5: Table S2). TAK-242 significantly attenuated the increase in lactate (Additional file 5: Table S2) and reduced the L/P ratio (Figure 3) in both cortex and medulla. However, glucose and pyruvate levels remained stable, and no effect of treatment was observed (Additional file 5: Table S2).

\section{Treatment with a TLR4 inhibitor reduced renal neutrophil} infiltration

In the vehicle- and TAK-242-treated groups, increased amounts of PMN cells were found in the glomeruli and the interstitium/peritubular capillaries compared with controls. This was confirmed by quantification of PMNs by using immunohistochemistry (Figure 5). Compared with vehicle, TAK-242 significantly reduced the number of PMNs in the interstitium $(P=0.01$, Figure 6A) but not in the glomerulus $(P=0.16$, Figure $6 \mathrm{~B})$.

\section{Sepsis-induced endothelial swelling and decreased} fenestration in the glomeruli were attenuated by a TLR4 inhibitor

To examine the glomerular effects of sepsis and subsequent treatment with a TLR4 inhibitor, electron microscopy was used. There was no difference in the degree of foot process effacement or in the thickness of the glomerular basement membrane (GBM) between the vehicle-treated $(232 \pm 29 \mathrm{~nm})$, TAK-242-treated (264 \pm $72 \mathrm{~nm}$ ), and sham (242 $\pm 9 \mathrm{~nm}$ ) groups (Figure 7). However, there was a difference in the ultrastructure of the endothelial cells in the glomerular capillaries. The endothelial cells of the vehicle-treated animals were more swollen $(P=0.03$, Figures $6 \mathrm{C}$ and 7$)$ and showed a tendency for decreased fenestration $(P=0.07$, Figures $6 \mathrm{D}$ 


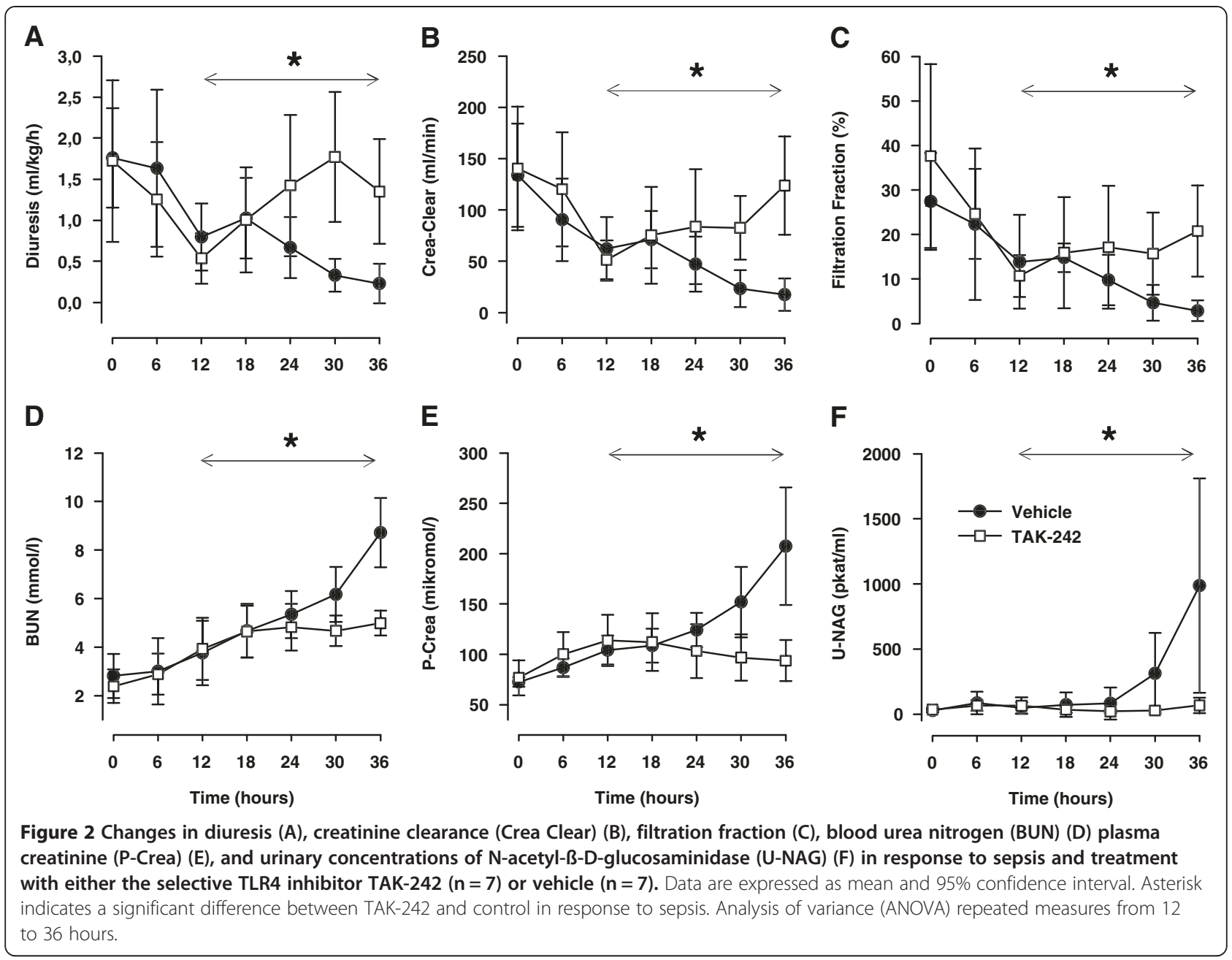

and 7) compared with TAK-242-treated animals and sham. Furthermore, the light microscopic observation of fibrin thrombi was confirmed in the two vehicle-treated animals (Additional file 4: Figure S1).

\section{TAK-242 had no effect per se}

There were no obvious differences in MAP, HR, CI, or MPAP between TAK-242 and vehicle during the 12hour treatment period in healthy sheep (Additional file 6: Figure S2). Renal function, as evaluated by creatinine clearance, plasma clearance, BUN, and urine output, remained stable, and no difference between treatments was seen (Additional file 7: Figure S3). Finally, renal blood flow and blood gas parameters remained unaffected by TAK-242 per se (Additional file 7: Figure S3 and Additional file 8: Table S4).

\section{Discussion}

In the present study, we aimed to investigate the renal effects of a TLR4-inhbitor, TAK-242, in experimental sepsis. The most striking finding was that TAK-242 reversed a progressive decline in renal function when administered therapeutically after 12 hours of hyperdynamic E. coli sepsis. This was associated with a prominent reduction in renal neutrophil infiltration, a decreased swelling of the endothelium in the glomerular capillaries, an attenuation of arterial and renal hyperlactemia, and less tubular damage as indicated by reduced U-NAG. Furthermore, no major effects on systemic or local renal hemodynamics by TAK-242 were seen during sepsis, and in separate experiments in animals without sepsis, TAK-242 had no obvious major effects per se.

Proper animal models of septic AKI are difficult to achieve, and too often they do not mimic the clinical scenario [24]. In this study, we used fluid-resuscitated, conscious animals developing severe hyperdynamic sepsis caused by live bacteria. Detailed renal functional data were collected repeatedly over an extended time period and related to immune function and histological pathology. In addition, treatment was started when sepsis and 


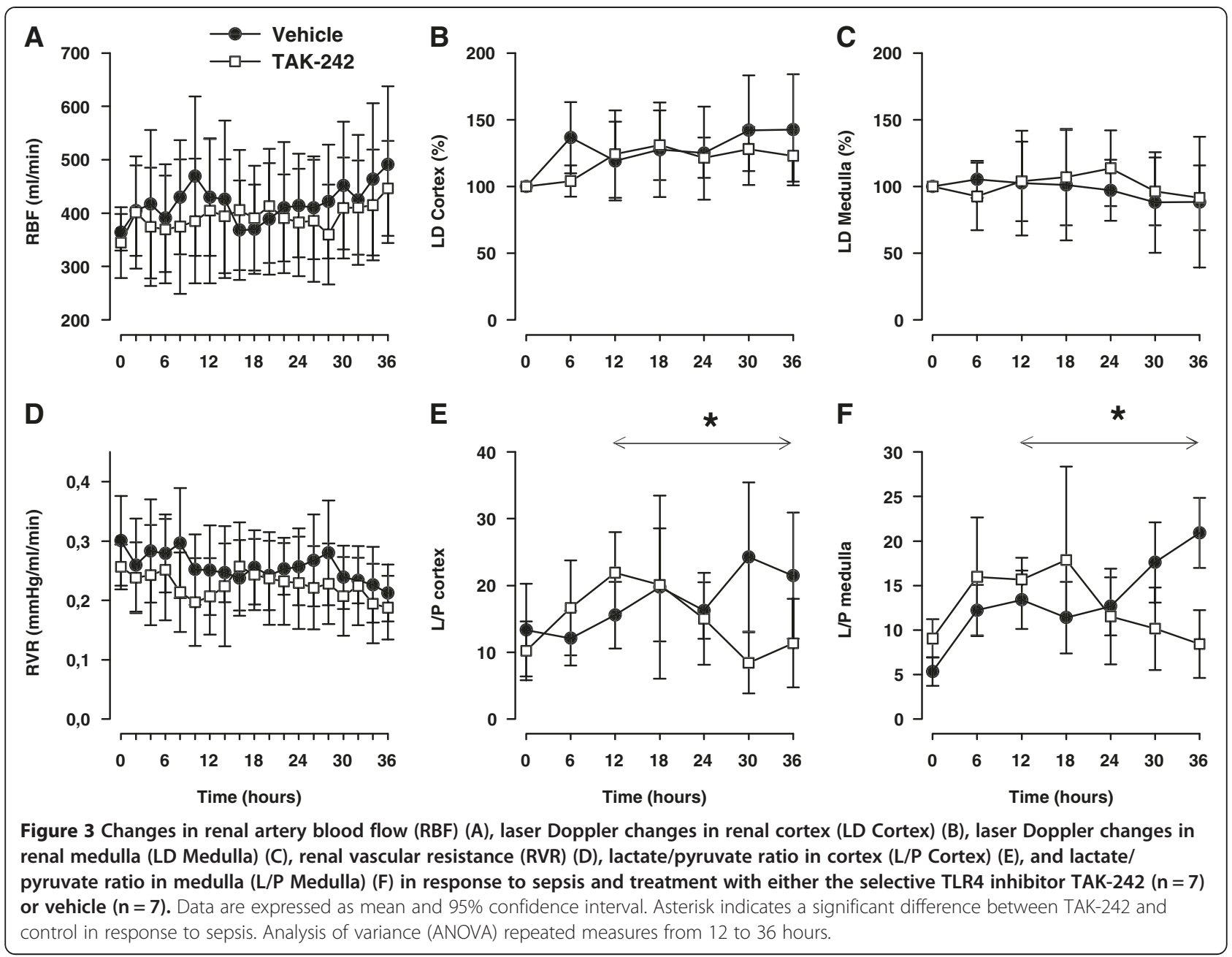

renal dysfunction were already present. Another strength of this investigation was that the renal histological findings in large mimicked what was recently described in human kidney after sepsis [6].

Innate immunity is the first line of defense against invading microbes and is crucial for preventing infections. TLR4 is central in this immune activation by binding LPS $[9,25]$, which results in an inflammatory response that induces the production and release of cytokines as well as stimulation of inflammatory cells [11]. Among these cells are neutrophils, which are activated and stimulated to transmigrate from blood to tissue. This is caused by TLR4-mediated release of interleukin-1B (IL-1B), tumor necrosis factor-alpha (TNF $\alpha$ ), and IL-6. Another important neutrophil activator released by TLR4 stimulation is serum amyloid protein 3 , which mediates its effect by targeting the formyl peptide receptor [26]. Although the inflammatory response is crucial for preventing infections, a strong activation of the innate immune system, like in sepsis, may inflict damage to endogenous tissue and impair organ function. TAK-242 binds selectively to the intracellular domain of TLR4 and disrupts the interaction with several adaptor molecules. This leads to inhibition of both the MyD88-dependent and the TRIF (TIR domain-containing adapter-inducing IFN- $\beta$ )-dependent pathway for TLR4 signal transduction [27].

Acute tubular necrosis (ATN) caused by ischemia has been assumed to be the underlying pathophysiology of septic AKI $[2,28]$. Massive release of NO is believed to cause vasodilation that result in relative hypovolemia, a reduction in cardiac output, and hypotension. As a reflex, renal sympathetic nerve activity is increased together with elevated levels of vasopressin, endothelin, angiotensin II, and aldosterone. The resulting renal vascular vasoconstriction leads to ischemia and subsequent ATN that impairs renal function. Undoubtedly, ischemia is a crucial factor for many types of AKI. However, in sepsis, the situation may sometimes be different. Most patients with sepsis present with a hyperdynamic circulation with elevated cardiac output [29]. Experimental 

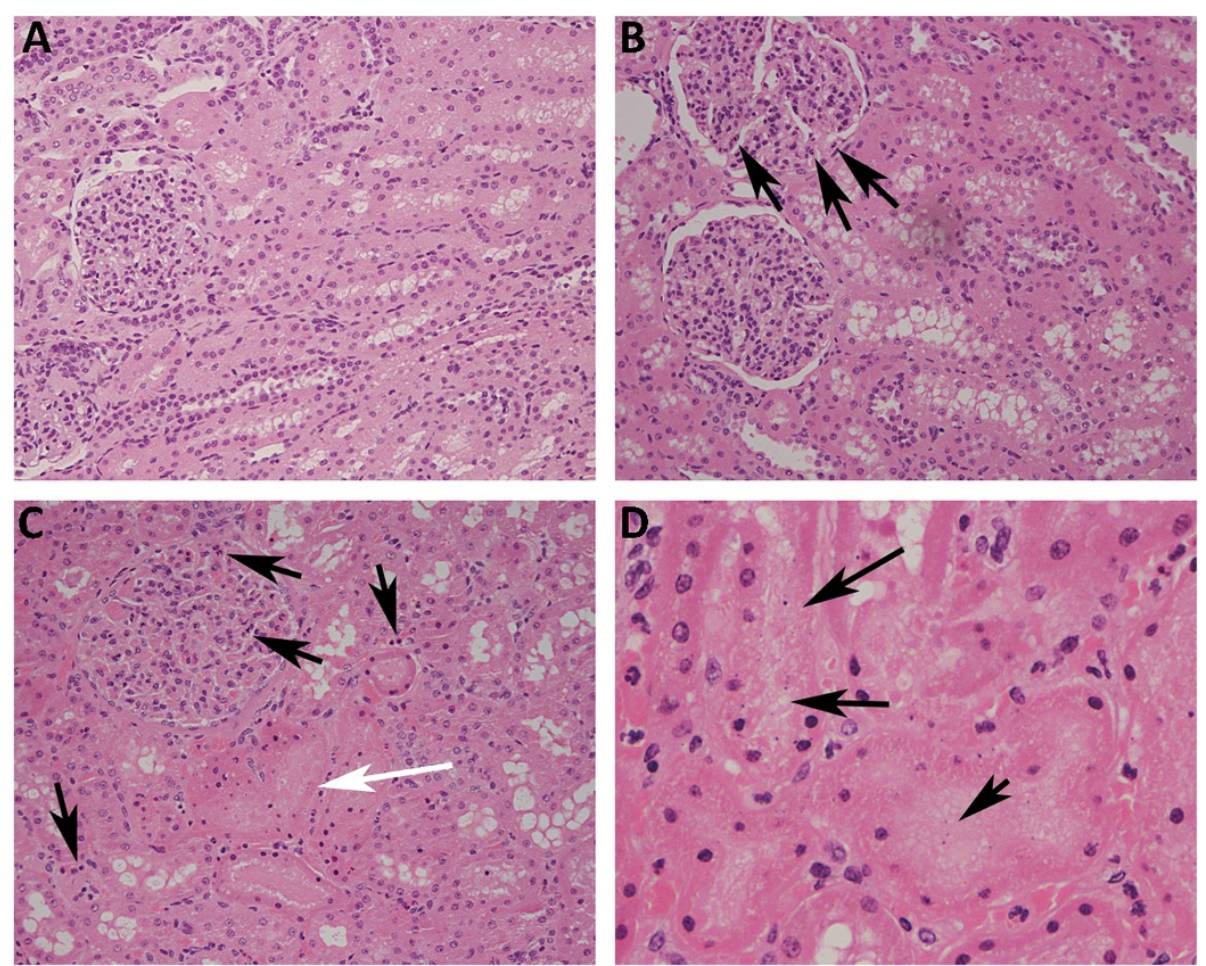

Figure 4 Increased renal neutrophil infiltration is attenuated by TLR4 inhibition. Light microscopy with hematoxylin/eosin staining shows an increased number of polymorphonuclear leukocytes (PMNs) (arrows) in the glomeruli and within the interstitium/peritubular capillaries in both vehicle-treated (C) and TAK-242-treated (B) animals in comparison with sham (A). Tubular epithelial injury was seen with pyknotic and disintegrated nuclei in two of the vehicle-treated animals - (C) white arrow and (D) arrows - and one of the TAK-242-treated animals.

data indicate that AKI develops although total renal blood flow is increased $[4,23]$ and hypotension is counteracted by vasoactive drugs [3]. Furthermore, the correlation between ATN and septic renal failure is weak in both human and animal studies [30]. The current results obtained in normotensive septic sheep confirm that hypotension is not a necessary attribute for septic AKI. This is in line with studies in critically ill patients with severe sepsis, in whom blood pressure levels did not correlate with the severity of renal failure [31]. TAK-242 drastically improved renal function without major hemodynamic effects. Moreover, there was no sign of renal vasoconstriction, as total renal artery blood flow as well as cortical and medullary perfusion did not decline during the 36-hour experiment. Thus, renal hypoperfusion appears highly unlikely as a cause of septic AKI, given this experimental setting. A recently promoted hypothesis for sepsis-induced AKI is that the inflammatory response causes a preferential dilatation of renal efferent arterioles, increasing renal blood flow but reducing the hydrostatic pressure for glomerular filtration and thereby glomerular filtration rate (GFR) [32]. The most important vasodilator released in sepsis is $\mathrm{NO}$, and it has been suggested that excessive intra-renal
NO could be responsible for the reduction in postglomerular resistance [33]. However, it is unlikely that the effect of TLR4 inhibition on renal function in this study is mediated by reduced NO formation as there were no differences in either MAP or renal blood flow and as TAK-242 did not affect NOx or cyclic guanosine monophosphate (cGMP) levels. This view is supported by results demonstrating no effect on renal function after intrarenal NO synthase inhibition during Gramnegative sepsis [33].

Both cortical and medullary ratios of $\mathrm{L} / \mathrm{P}$ were significantly increased by sepsis but subsequently reduced by TAK-242. In view of the preserved renal circulation, the renal hyperlactemia cannot easily be explained by reduced renal oxygen supply. However, direct measurement of renal tissue oxygenation or the utilization of oxygen by renal mitochondria was not performed in this study. Elevated lactate levels in sepsis not related to hypoxia have been linked to stimulation of muscle $\mathrm{Na} / \mathrm{K}-\mathrm{ATPase}[34]$ and mitochondrial dysfunction [35]. Thus, besides hypoxia, other factors may explain the renal hyperlactemia.

Sepsis caused severe endothelial swelling and decreased glomerular fenestration, both of which were reduced by 

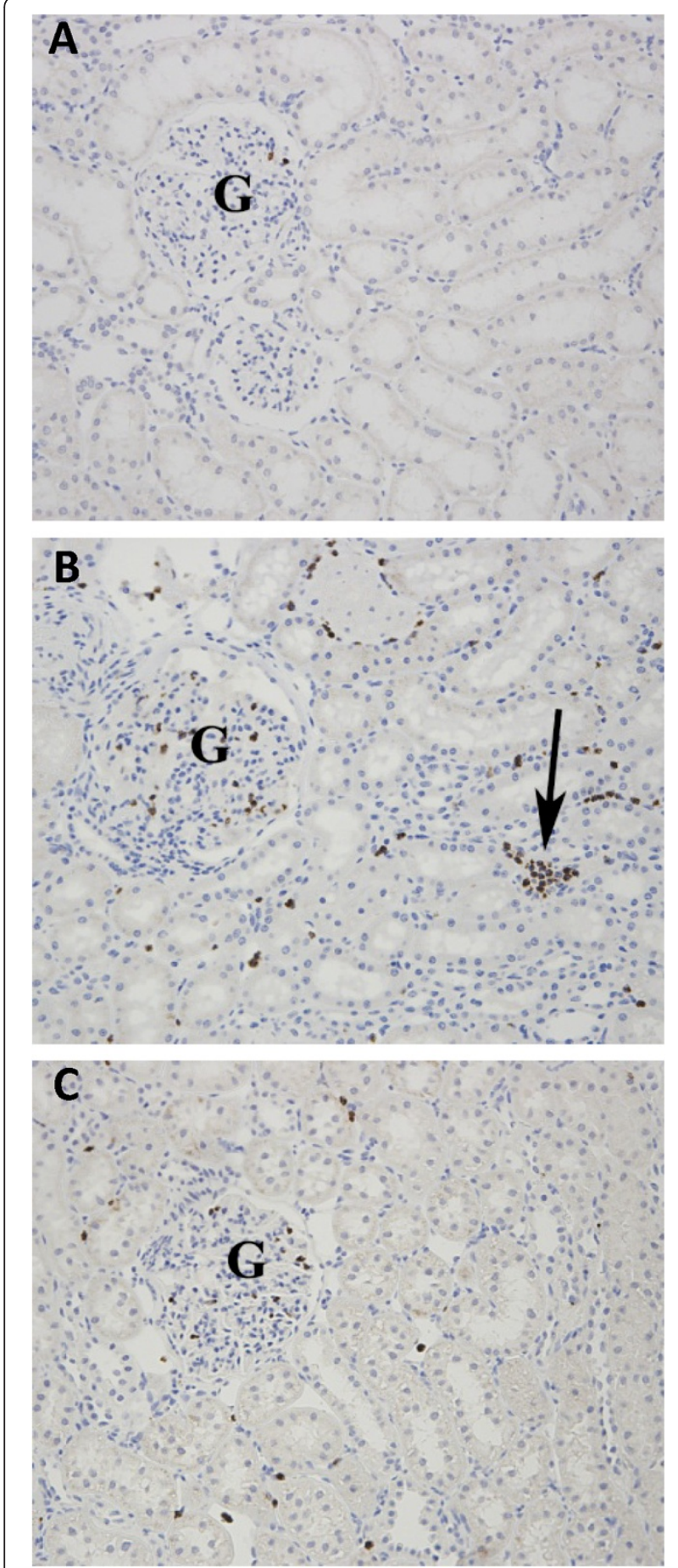

Figure 5 Immunohistochemistry with anti-myeloperoxidase antibodies show increased number of polymorphonuclear leukocytes (PMNs) in glomerular capillaries (G) and interstitium/ peritubular capillaries (arrow) in vehicle-treated (B) and TAK-242-treated (C) group in comparison with sham (A).

TLR4 inhibition. The renal dysfunction observed may, to a large extent, be a consequence of decreased glomerular filtration per se, as creatinine clearance and the filtration fraction decreased significantly in the vehicle-treated animals, without significant tubular damage. Endothelial dysfunction in the peritubular capillaries has been highlighted as an important injury pathway in septic AKI [36], possibly by inducing ATN [37]. Herein, U-NAG did increase in vehicle-treated animals as a possible sign of tubular injury, but this took place several hours after renal function started to decline and was not confirmed by light microscopy. Instead, it is possible that endothelial swelling and perhaps decreased fenestration in the glomerulus may play an important role in the injury pathway and propagation of septic AKI. However, an early and specific marker of glomerular membrane dysfunction is urine protein leakage, and this was not detected in this study and is not a hallmark of sepsis-induced AKI.

Mobilization and recruitment of PMNs by the innate immune defense constitute a key event in response to an infection. After extravasation, PMNs destroy invading organisms by phagocytosis, release of acid hydrolases and antimicrobial peptides, and stimulation of antibiotic actions of monocytes and macrophages [38]. However, PMN degranulation may also inflict damage to endogenous tissue. The role of PMNs in septic AKI is largely unknown, but after renal ischemia and reperfusion, PMNs have been shown to transmigrate from the circulation and contribute to AKI [39]. In sepsis, Castoldi and colleagues [40] recently showed that TLR4-deficient mice had reduced renal neutrophil activation and infiltration compared with wild-type mice and that neutrophil depletion improved renal function. This indicates that the extensive renal neutrophil accumulation caused by sepsis in this study is potentially deleterious. Activation of renal endothelial TLR4 has been suggested to play a critical role in the upregulation of adhesion molecules which can promote the recruitment of leukocytes to areas of injury and aggravate damage and inflammation in the tissue [41,42]. A TLR4-dependent pathway promoting renal injury and inflammation in antibodymediated glomeruli-nephritis and cisplatin-induced nephrotoxicity has also been described $[43,44]$. Therefore, reduced TLR4-dependent infiltration of PMNs may have contributed to the improved renal function in the TAK242 group. This is supported by recent findings of substantial renal leukocyte infiltration in human septic shock [6]. Possible mechanisms for the renal dysfunction include PMN vascular endothelial attachment that reduces blood flow and causes ischemia [45] or tubular damage by migrated PMNs [46]. However, as discussed previously, no rheological effects of sepsis or TLR4 inhibition were noted in the current study, and light microscopy revealed no convincing evidence of general or widely spread structural damage to the tubules. Taken together, these observations indicate that neutrophil activation and recruitment into the kidney are potential and perhaps crucial mediators of 

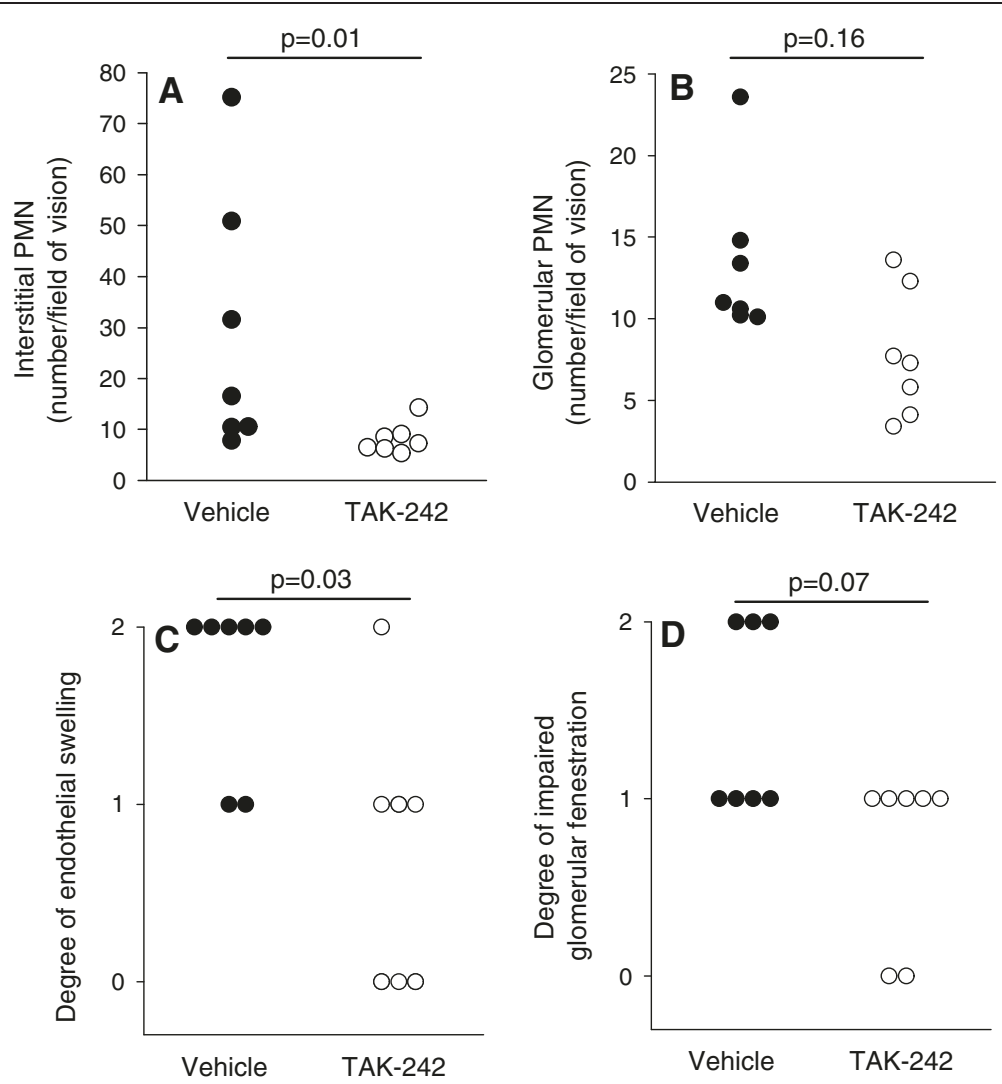

Figure 6 Number of polymorphonuclear leukocytes (PMNs) in interstitium (A) and glomerulus (B) as well as histological determination of endothelial swelling (C) and degree of fenestration in vehicle-treated (D) $(n=7)$ and TAK-242-treated $(n=7)$ animals. Zero indicates no swelling/decreased fenestration, 1 indicates some swelling/decreased fenestration, and 2 indicates severe swelling/decreased fenestration as determined by electron microscopy. Data are expressed as raw numbers. $P<0.05$ is considered significant.

septic AKI, although the underlying mechanism remains unknown. As shown in a series of elegant experiments by Watts and colleagues [47-49], TLR4 activation may also affect renal function by impairing tubular transport. In particular, $\mathrm{HCO}_{3}^{-}$reabsorption is inhibited by the direct effect of LPS on TLR4, and this may contribute to sepsisinduced acidosis.

Besides renal effects, TLR4 inhibition significantly attenuated the increase in mean pulmonary artery pressure and prevented the decrease in partial pressure of oxygen. The mechanism for these findings is unknown. However, a TLR4-dependent pathway for recruitment of neutrophils into the lung has been highlighted in endotoxemic mice [41], and PMN degranulation may cause severe lung damage [38]. The reason why arterial $\mathrm{pCO}_{2}$ did not change is unknown but may be related to the fact that $\mathrm{CO}_{2}$, compared with oxygen, more easily diffuses between blood and alveolus.

The current results are partly in contrast to the recent clinical trial using a TLR4 antagonist in sepsis $[19,20]$. In that study, no effect on survival was discovered. However, data on renal function were not reported, and only a minority of the patients had verified Gram-negative sepsis. Thus, it is possible that some patients with sepsis would still benefit from TLR4 inhibition.

We would like to acknowledge some of the limitations of this study. Evaluation of the renal microcirculation was performed with the use of laser Doppler probes surgically implanted in both the cortex and medulla. This is a wellestablished method and is frequently used for assessment of renal tissue perfusion; however, the technique is invasive, and measurements are performed at a small proportion of the kidney, less than approximately $1 \mathrm{~mm}^{3}$ at each probe site. Although the technique is a good option for continuous data acquisition [50], it has problems detecting heterogeneity in microvascular flow. The use of creatinine clearance as an indicator for GFR is often used in the clinical setting, but the accuracy is limited in patients. However, in sheep, renal clearance of endogenous creatinine may be a relatively adequate measure of GFR [51].

\section{Conclusions}

In summary, our data show that TLR4 signaling is important for the development of septic AKI and that treatment 

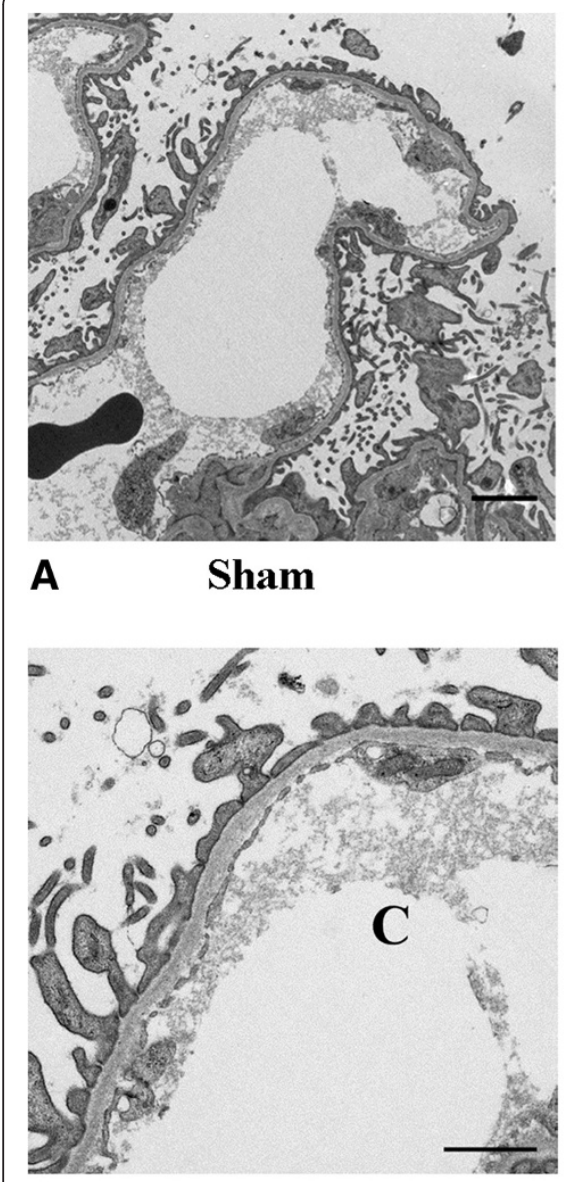

\section{Sham}

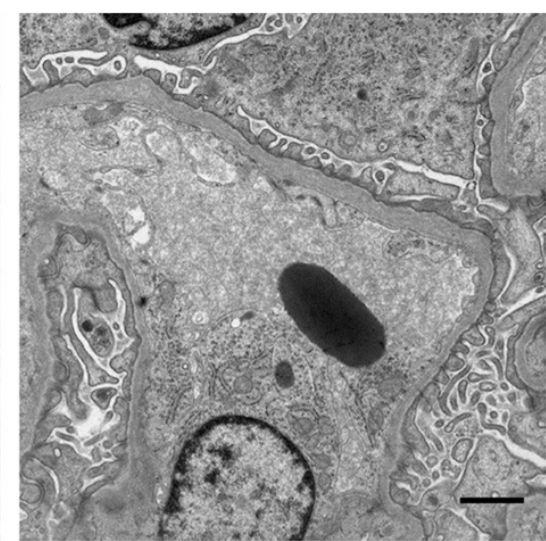

B

Vehicle

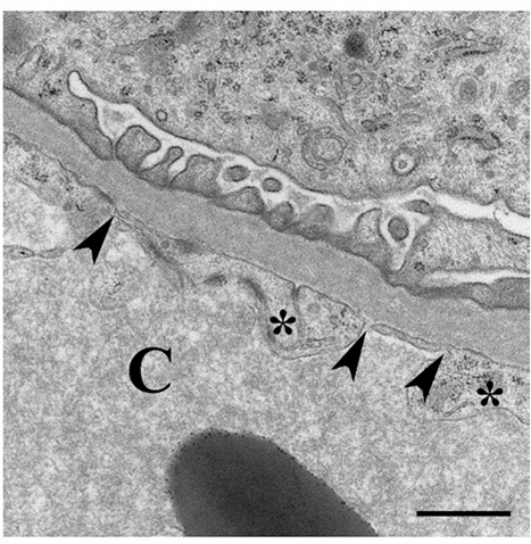

E Vehicle

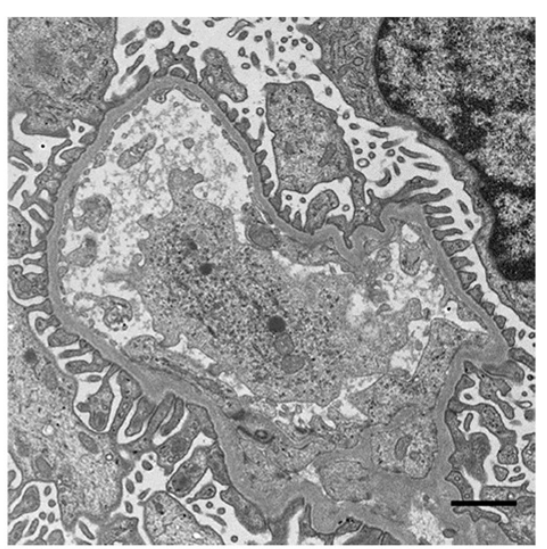

C

TAK-242

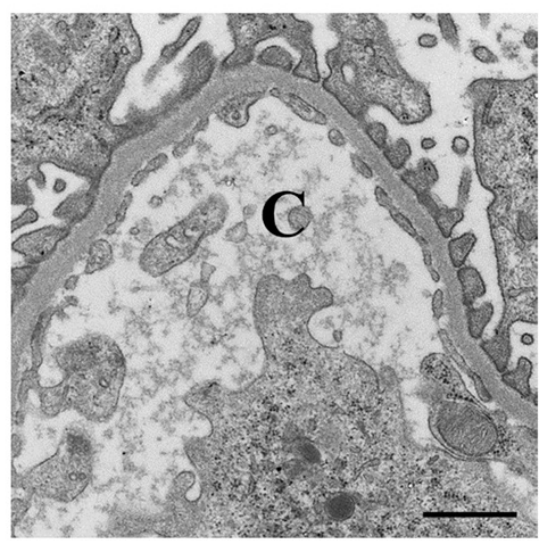

$\mathbf{F}$
TAK-242

Figure 7 Electron microscopic images of the glomerular capillaries in sham (A), vehicle-treated (B), and TAK-242-treated (C) animals. The same images magnified showing glomerular capillaries in sham (D), vehicle-treated (E), and TAK-242-treated (F) animals. Asterisk indicates endothelial cell swelling, and arrowheads show decreased fenestration of the glomerular endothelium. Bars $=2 \mu \mathrm{m}(\mathbf{A}, \mathbf{B}, \mathbf{C})$ and $1 \mu \mathrm{m}(\mathbf{D}, \mathbf{E}, \mathbf{F})$.

with a TLR4 inhibitor is able to reverse a manifest reduction in renal function caused by sepsis. Although further studies are necessary, this suggests that targeting TLR4 may be a potential way to treat AKI caused by Gramnegative sepsis. Furthermore, in the present model, septic AKI is not due to hypoperfusion-induced ischemia but rather an inflammatory reaction within the kidney reducing glomerular filtration. Endothelial swelling in the glomerulus may be a contributing factor for the latter.

\section{Key messages}

- Inhibiting Toll-like receptor 4 reverses a manifest renal dysfunction caused by $E$. coli sepsis.

- In the present model, septic acute kidney injury is not due to hypoperfusion-induced ischemia but rather an inflammatory reaction within the kidney.
- Endothelial swelling in the glomerulus may be a contributing factor for the reduced glomerular filtration.

- Toll-like receptor 4 antagonism has no obvious effects on renal function in healthy individuals.

\section{Additional files}

Additional file 1: Detailed Materials and Methods. A more extensive description of materials and methods.

Additional file 2: Table S3. Sham-operated animal without sepsis. Data are for sham animal without sepsis $(n=1)$ and expressed as raw data from baseline to 36 hours. Cl, cardiac index; cort, cortex; Crea clearance, creatinine clearance; $H R$, heart rate; Lactate ${ }_{\text {art }}$, arterial levels of lactate; LD, laser Doppler; L/P, Lactate/Pyruvate ratio; MAP, mean arterial pressure; med, medulla; MD, microdialysis; MPAP, mean pulmonary arterial pressure; $\mathrm{PCO}_{2}$, partial pressure of carbon dioxide; $\mathrm{pO}_{2}$, partial pressure of oxygen; P-Protein, plasma protein; RBF, renal blood flow. 
Additional file 3: Table S1. Systemic variables. Data are for TAK-242 and control expressed as mean and standard deviation (SD). Asterisk indicates a significant difference between TAK-242 and control in response to sepsis. Analysis of variance (ANOVA) repeated measures, including 12, 18, 24, 30, and 36 hours. Differences were considered significant at $P \leq 0.05$. cGMP, cyclic guanosine monophosphate; $\mathrm{NOx}$, the sum of nitrite and nitrate concentration; $\mathrm{pCO}_{2}$, partial pressure of carbon dioxide; $\mathrm{pO}_{2}$, partial pressure of oxygen; p-protein, plasma protein.

Additional file 4: Figure S1. Fibrin thrombi formation in glomerular capillaries. Fibrin thrombi formation in glomerular capillaries. In two of the vehicle-treated animals, fibrin thrombi (arrow) were seen both in light microscopy with Ladewig staining (a) and in electron microscopy (b).

Additional file 5: Table S2. Microdialysis in renal cortex and medulla. Data are for TAK-242 and control expressed as mean and standard deviation (SD). Asterisk indicates a significant difference between TAK-242 and control in response to sepsis. Analysis of variance (ANOVA) repeated measures, including $12,18,24,30$, and 36 hours. Differences were considered significant at $P \leq 0.05$. cort, cortex; MD, microdialysis; med, medulla.

Additional file 6: Figure S2. Changes in heart rate (A), cardiac index (B), mean pulmonary artery pressure (MPAP) (C), and mean arterial pressure (MAP) (D) in response to treatment with either the selective TLR4 inhibitor TAK-242 (filled symbols) or vehicle (open symbols). $N=3$ in a cross-over design. Data are shown for each individual animal.

Additional file 7: Figure S3. Changes in creatinine clearance (A), P-creatinine (B), blood urea nitrogen (BUN) (C), renal blood flow (D), and urine output (E) in response to treatment with either the selective TLR4 inhibitor TAK-242 (filled symbols) or vehicle (open symbols). $\mathrm{N}=3$ in a cross-over design. Data are shown for each individual animal.

Additional file 8: Table S4. Sheep without sepsis treated with TAK-242. Blood gas data for three healthy sheep receiving TAK-242 or vehicle for 12 hours in a cross-over design.

\section{Abbreviations}

AKI: acute kidney injury; ANOVA: analysis of variance; ATN: acute tubular necrosis; BUN: blood urea nitrogen; Cl: cardiac index; GBM: glomerular basement membrane; GFR: glomerular filtration rate; HR: heart rate; L/P: lactate/pyruvate; LPS: lipopolysaccharide; MAP: mean arterial blood pressure; MPAP: mean pulmonary arterial blood pressure; NAG: N-acetylbeta-D-glucosaminidas; NO: nitric oxide; NOx: sum of nitrite and nitrate; $\mathrm{pCO}_{2}$ : partial pressure of carbon dioxide; PMN: polymorphonuclear leukocyte; TLR4: Toll-like receptor 4.

\section{Competing interests}

The authors declare that they have no competing interests.

\section{Authors' contributions}

JoF conceived and designed the study, carried out the animal experiments, participated in the analyses of biological samples, performed the statistical analyses, and drafted the manuscript. MR carried out the animal experiments and participated in the analyses of biological samples. $\mathrm{KH}$ performed the electron microscopy investigation and participated in the analyses of data. $\mathrm{JaF}, \mathrm{MT}$, and SE participated in the animal experiments and contributed in drafting the manuscript. EK performed analyses of biological samples. EW contributed to the data analyses and analyzed biological samples. VO helped to design the study and supplied the bacteria to induce sepsis. AW performed the histology and participated in the analyses of data. RF conceived and designed the study, carried out the animal experiments, participated in the analyses of biological samples, and drafted the manuscript. All authors read and approved the final manuscript.

\section{Acknowledgments}

We are most grateful for the excellent laboratory assistance by Azar Baharpoor and Carina Nihlen, Department of Physiology and Pharmacology, Karolinska Institutet, Stockholm. Takeda Pharmaceutical Company Limited (Osaka, Japan) kindly provided us with TAK-242. The study was supported by funds from the Swedish Research Council (grant 521-2011-2843, RF), Karolinska University Hospital (JoF, JaF, AW, KH, AW, and VO), Karolinska Institutet (MR and SE), The Lars Hierta Foundation (JoF), The Swedish Society of
Medicine (RF), The Swedish Society for Anaesthesia and Intensive Care (JoF), The Ruth and Richard Juhlin Foundation (RF), The Åke Wiberg Foundation (RF), and The Swedish Kidney Association (JoF). There was no involvement of the funding resources in research design collection, analysis, and interpretation of data. The writing and publication of the manuscript were done by the authors without any participation or influence from the funding sources for any of the authors.

\section{Author details}

'Department of Anesthesia and Intensive Care, Karolinska University Hospital, Department of Clinical Science Intervention and Technology, Karolinska Institutet, Hälsovägen 13, 14157 Huddinge, Sweden. ${ }^{2}$ Department of Physiology and Pharmacology, Karolinska Institutet, von Eulers väg 8, 17177 Stockholm, Sweden. ${ }^{3}$ Department of Laboratory Medicine, Division of KFC, Karolinska Institutet, Hälsovägen 13, 14157 Huddinge, Sweden. ${ }^{4}$ Department of Surgical Sciences, Section of Anaesthesiology \& Intensive Care, Uppsala University, Sjukhusvägen 1, 75185 Uppsala, Sweden. ${ }^{5}$ Department of Molecular Medicine and Surgery, Karolinska Institutet, Karolinska vägen 1, 17176 Stockholm, Sweden. ${ }^{6}$ Center of Molecular Medicine, Karolinska University Hospital, Karolinska vägen 1, 17176 Stockholm, Sweden. ${ }^{7}$ Division of Clinical Microbiology, Karolinska Institutet, Karolinska University Hospital, Hälsovägen 13, 14157 Huddinge, Sweden. ${ }^{8}$ Department of Clinical Science Intervention and Technology, Division of Renal Medicine, Karolinska Institutet, Hälsovägen 13, 14157 Huddinge, Sweden.

Received: 18 March 2014 Accepted: 4 August 2014

Published online: 03 September 2014

\section{References}

1. Murugan R, Kellum JA: Acute kidney injury: what's the prognosis? Nat Rev Nephrol 2011, 7:209-217.

2. Schrier RW, Wang W: Acute renal failure and sepsis. N Engl J Med 2004, 351:159-169.

3. Fenhammar J, Rundgren M, Forestier J, Kalman S, Eriksson S, Frithiof R: Toll-like receptor 4 inhibitor TAK-242 attenuates acute kidney injury in endotoxemic sheep. Anesthesiology 2011, 114:1130-1137.

4. Frithiof R, Soehnlein O, Eriksson S, Fenhammar J, Hjelmqvist H, Lindbom L, Rundgren $\mathrm{M}$ : The effects of isoflurane anesthesia and mechanical ventilation on renal function during endotoxemia. Acta Anaesthesiol Scand 2011, 55:401-410.

5. Ishikawa K, Bellomo R, May CN: The impact of intrarenal nitric oxide synthase inhibition on renal blood flow and function in mild and severe hyperdynamic sepsis. Crit Care Med 2011, 39:770-776.

6. Lerolle N, Nochy D, Guerot E, Bruneval P, Fagon JY, Diehl JL, Hill G: Histopathology of septic shock induced acute kidney injury: apoptosis and leukocytic infiltration. Intensive Care Med 2010, 36:471-478.

7. Medzhitov R, Preston-Hurlburt P, Janeway CA Jr: A human homologue of the Drosophila Toll protein signals activation of adaptive immunity. Nature 1997, 388:394-397.

8. Hoshino K, Takeuchi O, Kawai T, Sanjo H, Ogawa T, Takeda Y, Takeda K, Akira S: Cutting edge: Toll-like receptor 4 (TLR4)-deficient mice are hyporesponsive to lipopolysaccharide: evidence for TLR4 as the Lps gene product. I Immunol 1999, 162:3749-3752.

9. Poltorak A, He X, Smirnova I, Liu MY, Van Huffel C, Du X, Birdwell D, Alejos E, Silva M, Galanos C, Freudenberg M, Ricciardi-Castagnoli P, Layton B, Beutler B: Defective LPS signaling in $\mathrm{C} 3 \mathrm{H} / \mathrm{HeJ}$ and $\mathrm{C} 57 \mathrm{BL} / 10 \mathrm{ScCr}$ mice: mutations in Tlr4 gene. Science 1998, 282:2085-2088.

10. Qureshi ST, Lariviere L, Leveque G, Clermont S, Moore KJ, Gros P, Malo D: Endotoxin-tolerant mice have mutations in Toll-like receptor 4 (TIr4). J Exp Med 1999, 189:615-625.

11. Kawai T, Akira S: TLR signaling. Semin Immunol 2007, 19:24-32.

12. Daubeuf B, Mathison J, Spiller S, Hugues S, Herren S, Ferlin W, Kosco-Vilbois M, Wagner H, Kirschning CJ, Ulevitch R, Elson G: TLR4/MD-2 monoclonal antibody therapy affords protection in experimental models of septic shock. J Immunol 2007, 179:6107-6114.

13. Goldfarb RD, Ortegel JW, Parrillo JE, Zanotti-Cavazzoni S, Casey LC, Dellinger RP: TAKEDA-143242 increased survival via reduced cytokines in porcine peritonitis. J Surg Res 2011, 166:e165-e173.

14. Kuno M, Nemoto K, Ninomiya N, Inagaki E, Kubota M, Matsumoto T, Yokota $\mathrm{H}$ : The novel selective toll-like receptor 4 signal transduction inhibitor tak-242 prevents endotoxaemia in conscious Guinea-pigs. Clin Exp Pharmacol Physiol 2009, 36:589-593. 
15. Roger T, Froidevaux C, Le Roy D, Reymond MK, Chanson AL, Mauri D, Burns K, Riederer BM, Akira S, Calandra T: Protection from lethal gram-negative bacterial sepsis by targeting Toll-like receptor 4. Proc Natl Acad Sci U S A 2009, 106:2348-2352.

16. Sha T, Sunamoto M, Kitazaki T, Sato J, li M, lizawa Y: Therapeutic effects of TAK-242, a novel selective Toll-like receptor 4 signal transduction inhibitor, in mouse endotoxin shock model. Eur J Pharmacol 2007, 571:231-239.

17. Sha T, lizawa Y, li M: Combination of imipenem and TAK-242, a Toll-like receptor 4 signal transduction inhibitor, improves survival in a murine model of polymicrobial sepsis. Shock 2011, 35:205-209.

18. Tidswell M, Tillis W, Larosa SP, Lynn M, Wittek AE, Kao R, Wheeler J, Gogate J, Opal SM: Phase 2 trial of eritoran tetrasodium (E5564), a toll-like receptor 4 antagonist, in patients with severe sepsis. Crit Care Med 2010, 38:72-83.

19. Opal SM, Laterre PF, Francois B, LaRosa SP, Angus DC, Mira JP, Wittebole X, Dugernier T, Perrotin D, Tidswell M, Jauregui L, Krell K, Pachl J, Takahashi T, Peckelsen C, Cordasco E, Chang CS, Oeyen S, Aikawa N, Maruyama T, Schein R, Kalil AC, Van Nuffelen M, Lynn M, Rossignol DP, Gogate J, Roberts MB, Wheeler JL, Vincent JL, ACCESS Study Group: Effect of eritoran, an antagonist of MD2-TLR4, on mortality in patients with severe sepsis: the ACCESS randomized trial. JAMA 2013, 309:1154-1162.

20. Rice TW, Wheeler AP, Bernard GR, Vincent $J$, Angus DC, Aikawa N, Demeyer I, Sainati S, Amlot N, Cao C, li M, Matsuda H, Mouri K, Cohen J: A randomized, double-blind, placebo-controlled trial of TAK-242 for the treatment of severe sepsis. Crit Care Med 2010, 38:1685-1694.

21. El Achkar TM, Huang X, Plotkin Z, Sandoval RM, Rhodes GJ, Dagher PC: Sepsis induces changes in the expression and distribution of Toll-like receptor 4 in the rat kidney. Am J Physiol Renal Physiol 2006, 290:F1034-F1043.

22. Cunningham PN, Wang Y, Guo R, He G, Quigg RJ: Role of Toll-like receptor 4 in endotoxin-induced acute renal failure. J Immuno/ 2004, 172:2629-2635.

23. Langenberg C, Wan L, Egi M, May CN, Bellomo R: Renal blood flow in experimental septic acute renal failure. Kidney Int 2006, 69:1996-2002.

24. Doi K, Leelahavanichkul A, Yuen PS, Star RA: Animal models of sepsis and sepsis-induced kidney injury. J Clin Invest 2009, 119:2868-2878.

25. Lemaitre B, Nicolas E, Michaut L, Reichhart JM, Hoffmann JA: The dorsoventral regulatory gene cassette spatzle/Toll/cactus controls the potent antifungal response in Drosophila adults. Cell 1996, 86:973-983.

26. Moreno G, Errea A, Van Maele L, Roberts R, Léger H, Sirard JC, Benecke A, Rumbo M, Hozbor D: Toll-like receptor 4 orchestrates neutrophil recruitment into airways during the first hours of Bordetella pertussis infection. Microbes Infect 2013, 15:708-718.

27. Matsunaga N, Tsuchimori N, Matsumoto T, li M: TAK-242 (resatorvid), a small-molecule inhibitor of Toll-like receptor (TLR) 4 signaling, binds selectively to TLR4 and interferes with interactions between TLR4 and its adaptor molecules. Mol Pharmacol 2011, 79:34-41.

28. Sharfuddin AA, Molitoris BA: Pathophysiology of ischemic acute kidney injury. Nat Rev Nephrol 2011, 7:189-200.

29. Flynn A, Chokkalingam MB, Mather PJ: Sepsis-induced cardiomyopathy: a review of pathophysiologic mechanisms. Heart Fail Rev 2010, 15:605-611

30. Langenberg C, Bagshaw SM, May CN, Bellomo R: The histopathology of septic acute kidney injury: a systematic review. Crit Care 2008, 12:R38.

31. Chawla LS, Seneff MG, Nelson DR, Williams M, Levy H, Kimmel PL, Macias WL: Elevated plasma concentrations of IL-6 and elevated APACHE II score predict acute kidney injury in patients with severe sepsis. Clin J Am SoC Nephrol 2007, 2:22-30.

32. May CN, Calzavacca P, Ishikawa K, Langenberg C, Wan L, Ramchandra R, Bellomo R: Novel targets for sepsis-induced kidney injury: the glomerular arterioles and the sympathetic nervous system. Exp Physiol 2012, 97:1168-1177.

33. Ishikawa K, Calzavacca P, Bellomo R, Bailey M, May CN: Effect of selective inhibition of renal inducible nitric oxide synthase on renal blood flow and function in experimental hyperdynamic sepsis. Crit Care Med 2012, 40:2368-2375

34. Levy B, Gibot S, Franck P, Cravoisy A, Bollaert PE: Relation between muscle $\mathrm{Na}+\mathrm{K}+$ ATPase activity and raised lactate concentrations in septic shock: a prospective study. Lancet 2005, 365:871-875.

35. Fredriksson K, Hammarqvist F, Strigard K, Hultenby K, Ljungqvist O, Wernerman J, Rooyackers O: Derangements in mitochondrial metabolism in intercostal and leg muscle of critically ill patients with sepsis-induced multiple organ failure. Am J Physiol Endocrinol Metab 2006, 291:E1044-E1050.
36. Wu L, Gokden N, Mayeux PR: Evidence for the role of reactive nitrogen species in polymicrobial sepsis-induced renal peritubular capillary dysfunction and tubular injury. J Am Soc Nephrol 2007, 18:1807-1815.

37. Sharfuddin AA, Sandoval RM, Berg DT, McDougal GE, Campos SB, Phillips $\mathrm{CL}$, Jones BE, Gupta A, Grinnell BW, Molitoris BA: Soluble thrombomodulin protects ischemic kidneys. J Am Soc Nephrol 2009, 20:524-534.

38. Soehnlein O, Oehmcke S, Ma X, Rothfuchs AG, Frithiof R, van Rooijen N, Mörgelin M, Herwald $H$, Lindbom L: Neutrophil degranulation mediates severe lung damage triggered by streptococcal M1 protein. Eur Respir J 2008, 32:405-412.

39. Awad AS, Rouse M, Huang L, Vergis AL, Reutershan J, Cathro HP, Linden J, Okusa MD: Compartmentalization of neutrophils in the kidney and lung following acute ischemic kidney injury. Kidney Int 2009, 75:689-698.

40. Castoldi A, Braga TT, Correa-Costa M, Aguiar CF, Bassi EJ, Correa-Silva R, Elias RM, Salvador F, Moraes-Vieira PM, Cenedeze MA, Reis MA, Hiyane MI, Pacheco-Silva A, Goncalves GM, Saraiva Camara NO: TLR2, TLR4 and the MYD88 signaling pathway are crucial for neutrophil migration in acute kidney injury induced by sepsis. PLoS One 2012, 7:e37584.

41. Andonegui $\mathrm{G}$, Bonder CS, Green F, Mullaly SC, Zbytnuik L, Raharjo E, Kubes P: Endothelium-derived Toll-like receptor-4 is the key molecule in LPS-induced neutrophil sequestration into lungs. J Clin Invest 2003, 111:1011-1020.

42. Chen J, John R, Richardson JA, Shelton JM, Zhou XJ, Wang Y, Wu QQ, Hartono JR, Winterberg PD, Lu CY: Toll-like receptor 4 regulates early endothelial activation during ischemic acute kidney injury. Kidney Int 2011, 79:288-299.

43. Brown HJ, Lock HR, Wolfs TG, Buurman WA, Sacks SH, Robson MG: Toll-like receptor 4 ligation on intrinsic renal cells contributes to the induction of antibody-mediated glomerulonephritis via CXCL1 and CXCL2. J Am SoC Nephrol 2007, 18:1732-1739.

44. Zhang B, Ramesh G, Uematsu S, Akira S, Reeves WB: TLR4 signaling mediates inflammation and tissue injury in nephrotoxicity. J Am SoC Nephrol 2008, 19:923-932.

45. Molitoris BA, Sandoval RM: Intravital multiphoton microscopy of dynamic renal processes. Am J Physiol Renal Physiol 2005, 288:F1084-F1089.

46. Klausner JM, Paterson IS, Goldman G, Kobzik L, Rodzen C, Lawrence R, Valeri CR, Shepro D, Hechtman HB: Postischemic renal injury is mediated by neutrophils and leukotrienes. Am J Physiol 1989, 256:F794-F802.

47. Watts BA III, George T, Sherwood ER, Good DW: Basolateral LPS inhibits NHE3 and HCOFormula absorption through TLR4/MyD88-dependent ERK activation in medullary thick ascending limb. Am J Physiol Cell Physiol 2011, 301:C1296-C1306.

48. Watts BA III, George T, Good DW: Lumen LPS inhibits HCO3(-) absorption in the medullary thick ascending limb through TLR4-PI3K-Akt-mTOR-dependent inhibition of basolateral $\mathrm{Na}+/ \mathrm{H}+$ exchange. Am J Physiol Renal Physiol 2013, 305:F451-F462.

49. Watts BA III, George T, Sherwood ER, Good DW: A two-hit mechanism for sepsis-induced impairment of renal tubule function. Am J Physiol Renal Physiol 2013, 304:F863-F874.

50. Sullivan JC, Wang B, Boesen El, D'Angelo G, Pollock JS, Pollock DM: Novel use of ultrasound to examine regional blood flow in the mouse kidney. Am J Physiol Renal Physiol 2009, 297:F228-F235.

51. Nawaz M, Shah BH: Renal clearance of endogenous creatinine and urea in sheep during summer and winter. Res Vet Sci 1984, 36:220-224.

doi:10.1186/s13054-014-0488-y

Cite this article as: Fenhammar et al:: Renal effects of treatment with a TLR4 inhibitor in conscious septic sheep. Critical Care 2014 18:488. 